\title{
Erratum to: Determination of the Helical-Gear Geometry Taking Account of Total Teeth Wear
}

\author{
A. G. Popovich* \\ Zaporozhe Polytechnic, Zaporozhe, Ukraine \\ *e-mail: machinesmechanics@gmail.com \\ Received April 20, 2021; revised April 20, 2021; accepted April 20, 2021
}

DOI: $10.3103 / \mathrm{S} 1068798 X 21050270$

Page 987, left column, second paragraph, in the last sentence, $x_{D 1}=0.6472$ is considered true (instead of $x_{D 1}=0.6742$ ).

Page 987, left column, fourth paragraph, in the third sentence, $0.3016>x_{2}>0.2238$ is considered true (instead of $0.3061<x_{2}<0.2238$ ).

Page 987, left column, fourth paragraph, in the fourth sentence, $x_{2}=0.3016$ is considered true (instead of $x_{2}=$ $0.2238)$

The original article can be found online at

https://doi.org/10.3103/S1068798X20120138 